KANAZAWA-94-22

November, 1994

\title{
Radiative Symmetry Breaking in a Supersymmetric Model with an Extra $U(1)$
}

\author{
Daijiro Suematsu 因 \\ and \\ Yoshio Yamagishi 0 \\ Department of Physics, Kanazawa University, \\ Kanazawa 920-11, Japan
}

\begin{abstract}
Radiative symmetry breaking is studied in a superstring-inspired supersymmetric model which is extended with a low energy extra $U(1)$ symmetry. In this model the $\mu$-problem is radiatively solved in an automatic way. The right-handed neutrino can be heavy and the seesaw mechanism will produce the small neutrino mass which makes the MSW solution applicable to the solar neutrino problem. We search a parameter region which has the favorable feature for the radiative symmetry breaking at the weak scale. Rather wide parameter region is found to be allowed. The upper bound of the extra $Z$ boson mass is estimated as $M_{Z_{2}} \leq 2000 \mathrm{GeV}$ for a top mass range $150 \mathrm{GeV} \leq m_{t} \leq 190 \mathrm{GeV}$. Some phenomenological features of the extra $Z$ boson are also presented.
\end{abstract}

\footnotetext{
*e-mail:suematsu@hep.s.kanazawa-u.ac.jp

$\dagger$ †-mail:yamagisi@hep.s.kanazawa-u.ac.jp
} 


\section{Introduction}

Recently the supersymmetric theory attracts much attention. The analyses of the precise measurements of parameters in the standard model at LEP show that the gauge coupling unification occurs in a precise way in the minimal supersymmetric standard model(MSSM)[目]. These analyses also suggest that the top quark is very heavy $(\sim$ $162 \mathrm{GeV}$ [2]. CDF also reports that the top quark has been found at $m_{t}=174 \pm 16 \mathrm{GeV}[3]$. The heavy top quark is preferable to the radiative $S U(2) \times U(1)$ breaking in the supersymmetric model as pointed out more than ten years ago 4 . These facts seem to make the supersymmetric model, especially the MSSM, more attractive than before as a particle physics model.

However, the MSSM has some unsatisfactory features. Here we would like to stress two of them. The first one is a hierarchy problem in relation to the symmetry breaking, which is known as the $\mu$-problem[5]. The MSSM has a supersymmetric Higgs mixing term $\mu \bar{H} H$. To cause an appropriate radiative symmetry breaking at the weak scale we should put $\mu \sim O\left(G_{F}^{-1 / 2}\right)$ by hand, where $G_{F}$ is Fermi constant. Generally, in the supersymmetric model its typical scale is characterized by the supersymmetry breaking scale $M_{S}$. There is no reason why $\mu$ should be such a scale because it is irrelevant to the supersymmetry breaking. The reasonable way to answer this issue is to consider the origin of the $\mu$ scale as a result of the supersymmetry breaking. One of such solutions is the introduction of a singlet field $S$ and to replace $\mu \bar{H} H$ by a Yukawa type coupling $\lambda S \bar{H} H$. If $S$ gets a vacuum expectation value(VEV) of order $1 \mathrm{TeV}$ as a result of the soft supersymmetry breaking effect, $\mu \sim O\left(G_{F}^{-1 / 2}\right)$ will be realized dynamically as $\mu=\lambda\langle S\rangle$. A lot of works have been done on this type of models, in which the superpotential contains the terms $\lambda S \bar{H} H+\kappa S^{3}[5,8,9]$. From such studies it is known that the radiative symmetry breaking can occur successfully in a certain parameter region.

The second one is that there is no explanation for small neutrino masses in the MSSM framework. All known observations of the neutrino flux from the sun imply a deficit from the value predicted by the standard solar model [10]. It is very likely that these phenomena

\footnotetext{
${ }^{1}$ There are other solutions than the present one for the $\mu$-problem[6, []. However, in the models with extra gauge symmetries in the observable sector as the $E_{6}$ models, other solutions do not work so easily because it is difficult to construct a necessary term in the gauge invariant way. In this paper we will not consider them.
} 
are explained by the new neutrino properties. In order to introduce the small neutrino masses which can give a suitable solution of the solar neutrino problem, the MSSM must be extended in a certain way.

The most consistent supersymmetric model including the gravity is considered to be the superstring model[11]. There is a lot of progress in this model but the $N=1$ supergravity model can not be constructed as the satisfactory low energy effective theory of superstring still now. However, it has been shown by many efforts that its low energy gauge structure often contains extra gauge groups other than $S U(3) \times S U(2) \times U(1)$, especially, the extra $U(1) \mathrm{s}[12,13]$. These extra $U(1)$ symmetries should also be broken due to some dynamical mechanisms because in these models the mass scale other than the Planck scale $M_{\mathrm{pl}}$ is introduced only through the soft supersymmetry breaking. These breaking scales of extra $U(1)$ s induced by the $S U(3) \times S U(2) \times U(1)$ singlet fields may be relevant to the above mentioned problems. The low energy extra $U(1)$ may be related to the dynamical $\mu$-scale origin. The existence of the extra $U(1)$ is also convenient for the introduction of an intermediate scale without the large scale supersymmetry breaking. Such an intermediate scale may make neutrino masses small in a very simple mechanism. These aspects seem to make the extra $U(1)$ model very attractive as an extension of the MSSM.

In these extra $U(1)$ models it will be an important issue to examine the possibility of the radiative symmetry breaking of the low energy extra $U(1)$ and also their phenomenological features. In particular, from the phenomenological point of view it is interesting to study the mass bound of the extra $U(1)$ gauge boson in the recently suggested top quark mass region. These studies will be also of benefit to the superstring model building. In this paper we will investigate these issues on the basis of the renormalization group equations(RGEs).

This paper is organized as follows. In section 2 we define our model and discuss its features. We stress in what way the extra $U(1)$ s can solve the above mentioned problems in our model. In section 3 the scalar potential is analyzed to examine the symmetry breaking at the weak scale by using the RGEs. From such a study the various mass bounds of the physical particles are discussed. The section 4 is devoted to the summary. 


\section{An extra $U(1)$ model}

We consider a three generation rank-six model which is expected to derived from the superstring-inspired $E_{6}$ model. There are various types of such models. However, the model which can resolve the previously mentioned problems of the MSSM seems to be strongly restricted. The gauge structure of our model is $S U(3) \times S U(2) \times U(1)^{3}$. There are two extra $U(1)$ s. One will be related to the $\mu$-problem and the other one will be essential for the explanation of the small neutrino mass. The massless field ingredients are summarized schematically as $3 \cdot \mathbf{2 7}+\delta \cdot(\Phi+\bar{\Phi})$, where $\mathbf{2 7}$ stands for a fundamental representation of $E_{6}$ and it contains the full one generation structure as is well-known. $\Phi$ and $\bar{\Phi}$ is some components of $\mathbf{2 7}$ and $\overline{\mathbf{2 7}}$, respectively. $\delta$ is a positive integer representing the multiplicity. Although the realization of the models with $\delta \neq 0$ is nontrivial, the existence of such solutions is known, for example, in the Calabi-Yau compactification[12, 13]. The concrete field assignments are presented in Table 1. Here it should be noted that these field assignments are different from those of the usually considered $E_{6}$ models with respect to the Higgs doublets, the color triplets and the singlet fields. A superpotential $W$ of this model is assumed to be expressed as

$W=h_{u}^{a b c} Q_{a} \bar{U}_{b} H_{c}+h_{d}^{a b c} Q_{a} \bar{D}_{b} \bar{H}_{c}+h_{\nu}^{a b c} L_{a} H_{b} N_{c}+h_{e}^{a b c} L_{a} \bar{H}_{b} \bar{E}_{c}+\lambda^{a b c} S_{a} \bar{H}_{b} H_{c}+k^{a b c} S_{a} g_{b} \bar{g}_{c}$,

where $a, b, c(=1 \sim 3)$ are the generation indices. As discussed later, one of the extra $U(1)$ symmetries $\left(U(1)_{y_{E}}\right)$ is considered to remain unbroken until the $O(1 \mathrm{TeV})$. Since in our field assignments the singlet field $S$ has a non-zero charge of this low energy extra $U(1)$ which will be clarified to be relevant to the $\mu$-problem later, the extra isosinglet colored fields $g$ and $\bar{g}$ can not be superheavy and remain massless until the low energy region in general. For the proton stability these extra color triplets $g$ and $\bar{g}$ should be assumed to decouple from the MSSM contents in the superpotential $W$ due to some discrete symmetries [12, 13]. Such symmetries can be introduced and are usually expected to exist in the superstring-inspired models.2]

Now we shall point out various features related to the extra $U(1)$ s of our model. First

\footnotetext{
${ }^{2}$ Here we assume the complete decoupling of $g$ and $\bar{g}$ in eq.(1), for simplicity. However, in order to prohibit the fast proton decay it is not necessary to impose such a strong conditions. There are many works on this problem. Recently in ref. [14 such a possibility is discussed in some details based on the discrete gauge anomaly cancellation.
} 
of all we show how the neutrinos can get the small masses. Here we confine ourselves to the one generation case for simplicity. The extension to the three generation case is straightforward. As suggested in the certain superstring models 12, 13, two kinds of massless neutrino-like chiral superfields can exist. One appears in pairs as $(\mathcal{N}, \overline{\mathcal{N}})$ which comes from $(\Phi+\bar{\Phi})$. One should note that $\overline{\mathcal{N}}$ is also the chiral superfield with the opposite charge to that of $\mathcal{N}$. For the other one we use the notation $N$ to represent it. It belongs to $3 \cdot \mathbf{2 7}$ and appears without a complex conjugate partner. Its fermionic component is recognized as a right-handed neutrino.

The pair of the chiral superfields $(\mathcal{N}, \overline{\mathcal{N}})$ has an extra $U(1)$ D-term flat direction $\langle\mathcal{N}\rangle=\langle\overline{\mathcal{N}}\rangle \equiv u$ because the D-term scalar potential for $(\mathcal{N}, \overline{\mathcal{N}})$ is proportional to $g_{E^{\prime}}^{2}\left(|\mathcal{N}|^{2}-|\overline{\mathcal{N}}|^{2}\right)^{2}$. As a result, $u$ is allowed to be an intermediate scale without breaking the supersymmetry at that scale 12]. The existence of the extra $U(1)$ prohibits the renormalizable terms for their self-interaction in the superpotential so that the superpotential for them contains only the nonrenormalizable terms. We assume that the lowest nonrenormalizable terms of the superpotential for these fields have the following form due to a certain discrete symmetry

$$
W=\frac{c_{0}}{M_{\mathrm{pl}}} \overline{\mathcal{N}}^{2} N^{2}+\frac{c_{n}}{M_{\mathrm{pl}}^{2 n-3}}(\mathcal{N} \overline{\mathcal{N}})^{n}
$$

where $n$ is an integer such as $n \geq 2\left[15\right.$. $c_{0}$ and $c_{n}$ are some constants. The scale of $u$ is determined by the minimization of the scalar potential derived from eq.(2), [3]

$$
V=c_{n}^{2} \frac{u^{4 n-2}}{M_{\mathrm{pl}}^{4 n-6}}-M_{N}^{2} u^{2} .
$$

$M_{N}$ is the soft breaking mass of $N$ and is assumed to be $O(1) \mathrm{TeV}$. This negative mass squared may be expected to be induced due to the special modular weight of $\mathcal{N}[16]$ and/or a radiative effect[17]. From eq.(3) the minimum of $V$ is found to be realized at the intermediate scale $u \sim\left(c_{n}^{-2} M_{\mathrm{pl}}^{4 n-6} M_{N}^{2}\right)^{\frac{1}{4 n-4}}$. Once these fields get such a VEV, the right-handed Majorana neutrino mass is produced due to the first term of eq.(2) as

$$
M_{R} \sim c_{0}\left(c_{n}^{-1} M_{\mathrm{pl}}^{n-2} M_{S}\right)^{\frac{1}{n-1}} .
$$

\footnotetext{
${ }^{3}$ The supergravity correction to the scalar potential is sufficiently suppressed by the inverse powers of $M_{\mathrm{pl}}$ so that the scalar potential can be reduced to the global supersymmetric one in the present case.
} 
The mass matrix of the neutrino sector is written on the basis $\left(L^{0}, N\right)$ as

$$
\mathcal{M}=\left(\begin{array}{cc}
0 & h_{\nu}\langle H\rangle \\
h_{\nu}\langle H\rangle & M_{R}
\end{array}\right)
$$

and the remaining neutral fermions completely decouple from these fields. Thus this mass matrix $\mathcal{M}$ can present the sufficiently small Majorana neutrino mass due to the seesaw mechanism as [18]

$$
m_{\nu} \sim \frac{\left(h_{\nu}\langle H\rangle\right)^{2}}{c_{0}\left(c_{n}^{-1} M_{\mathrm{pl}}^{n-2} M_{S}\right)^{\frac{1}{n-1}}} .
$$

As a typical example, let us take $h_{\nu}\langle H\rangle \sim 1 \mathrm{GeV}$ as the quark sector and put $c_{0}=$ $c_{n}=O(1)$ and $n=3$. For such values we get $u \sim 10^{15} \mathrm{GeV}$ and then $M_{R} \sim 10^{11} \mathrm{GeV}$. This induces $m_{\nu} \sim 10^{-2} \mathrm{eV}$, which is suitable to the MSW solution for the solar neutrino problem 19. This mechanism shows that the intermediate extra $U(1)$ is benefit for the explanation of small neutrino masses.

On the other hand a low energy extra $U(1)$ symmetry can play an important role to solve the $\mu$-problem. Similar model which is often called next-to-MSSM(NMSSM) [5, 8, 9] also contains a singlet Higgs $S$. In this type of model the $\kappa S^{3}$ term in the superpotential prepares the quartic coupling for the scalar component of $S$ and also prohibits a massless axion. The similar role is played by the extra $U(1)$ in the present model. The D-term of this extra $U(1)$ supplies the quartic coupling for the singlet $S$ and then guarantees the vacuum stability for $S$. The axion problem also disappears because of the existence of this extra $U(1)$ gauge symmetry. The detailed study of the $\mu$-problem needs the numerical analysis of the RGEs and it will be the main subject in the next section.

We should also comment on the CP phases in the soft supersymmetry breaking terms. In the present model the $\mu$-term is replaced by the Yukawa type interaction term. Then the corresponding soft supersymmetry breaking term becomes the ordinary A-term. This may be thought as one of the preferable features of our model because in such a case the physical $\mathrm{CP}$ phases in the soft supersymmetry breaking terms which contribute to the neutron electric dipole moment can be sufficiently suppressed in an automatic way if the origin of the supersymmetry breaking satisfies the rather suitable 16. This is based on the following mechanism that the phase structure of the $A$-term is similar to the one of the gaugino mass $M_{a}$ so that the fortunate cancellation can occur in the physical soft CP

\footnotetext{
${ }^{4}$ The similar mechanism is proposed in refs. 20.
} 
phase $\arg \left(A M_{a}^{*}\right)$. However, the usual soft breaking $B$-term corresponding to the $\mu$-term in the MSSM has no such property and its CP phase $\arg \left(B M_{a}^{*}\right)$ is very dangerous for the neutron electric dipole moment.

As is obvious from the previous discussion, the largest deviation of our model from the MSSM at the low energy region will be seen in the neutral current sector and also in the neutrino sector. Here let us briefly review the structure of the neutral gauge sector containing an extra $U(1)$ and comment on the lower bound of the VEV of $S$. From the various phenomenological reason we assume that the Higgs fields $\bar{H}_{3}, H_{3}$ and $S_{3}$ in the third generation alone get the VEVs as

$$
\left\langle\bar{H}_{3}\right\rangle=\left(\begin{array}{c}
\bar{v} \\
0
\end{array}\right), \quad\left\langle H_{3}\right\rangle=\left(\begin{array}{l}
0 \\
v
\end{array}\right), \quad\left\langle S_{3}\right\rangle=x
$$

and the VEVs of Higgs fields in the remaining generations vanish. For simplicity, every VEV is assumed to be real and positive. Here the VEV $x$ will be severely constrained by the experimental results for the neutral current. Putting $m_{Z}^{2}=\frac{1}{2}\left(g_{2}^{2}+g_{1}^{2}\right)\left(\bar{v}^{2}+v^{2}\right)$, the mass matrix of the neutral gauge bosons is expressed using the charge assignments in Table 1 as 22, 24]

$$
m_{Z}^{2}\left(\begin{array}{cc}
1 & 2 \eta \sin \theta_{W} \\
2 \eta \sin \theta_{W} & 4 \frac{\left(\sin \theta_{W}\right)^{2}}{\bar{v}^{2}+v^{2}}\left(\frac{3}{8} \bar{v}^{2}+\frac{1}{6} v^{2}+\frac{25}{24} x^{2}\right)
\end{array}\right) \equiv m_{Z}^{2}\left(\begin{array}{ll}
1 & b \\
b & a
\end{array}\right),
$$

where $\eta=\left(\frac{1}{\sqrt{6}} v^{2}-\frac{3}{2 \sqrt{6}} \bar{v}^{2}\right) /\left(\bar{v}^{2}+v^{2}\right)$. The mass eigenstates are defined by using a mixing angle $\theta$ as

$$
\left(\begin{array}{c}
Z_{1} \\
Z_{2}
\end{array}\right)=\left(\begin{array}{cc}
\cos \theta & \sin \theta \\
-\sin \theta & \cos \theta
\end{array}\right)\left(\begin{array}{c}
Z \\
Z^{\prime}
\end{array}\right)
$$

where $\theta$ is expressed as

$$
\tan ^{2} \theta=\frac{m_{Z}^{2}-m_{Z_{1}}^{2}}{m_{Z_{2}}^{2}-m_{Z}^{2}}
$$

The mass eigenvalues $m_{Z_{1}}^{2}$ and $m_{Z_{2}}^{2}$ of eq.(8) are

$$
m_{Z_{1,(2)}}^{2}=\frac{1}{2} m_{Z}^{2}\left[(1+a)-(+) \sqrt{(1-a)^{2}+4 b^{2}}\right] .
$$

${ }^{5}$ These features on the soft CP phases are also discussed based on the different mechanism in ref. 21].

${ }^{6}$ There is generally one more free parameter which represents a mixing effect. However, we shall neglect it in the present analysis [23] 
A lighter mass eigenstate $Z_{1}$ should be the observed $Z^{0}$ and $m_{Z_{1}}^{2} \cong m_{Z}^{2}$. From eq.(11) we find that this requires $a \gg 1, b$ or $x \gg \bar{v}, v$.

In order to restrict phenomenologically the value of $x$ in the stringent way, we refer to the neutral current data and others. For such a purpose it is convenient to draw the contours of the mass eigenvalue $m_{Z_{2}}$ and the mixing angle $\theta$ in the $(\bar{v} / v)-(x / v)$ plane. In Fig.1 we summarize such contours. In addition, generally there can be two different definition of the Weinberg angle as $\sin ^{2} \theta_{W} \equiv \frac{g_{1}^{2}}{g_{2}^{2}+g_{1}^{2}}$ and $\sin ^{2} \bar{\theta}_{W} \equiv 1-\frac{m_{W}^{2}}{m_{Z_{1}}^{2}}$, which are exactly equivalent in the MSSM limit $\left(m_{Z_{1}}^{2} \rightarrow m_{Z}^{2}\right)$ at tree level. As is well-known the radiative correction shifts them differently. The existence of the extra $U(1)$ makes them the different quantities even at the tree level so that after carefully subtracting the radiative correction effect $\Delta \equiv \sin ^{2} \theta_{W}-\sin ^{2} \bar{\theta}_{W}$ will be treated as the suitable measure for its existence. As a reference, we also draw the contours of $\Delta$ in Fig.1. By applying the experimental results to Fig.1 we may fairly restrict the values of VEVs. For example, the recently published constraint 25] on the mixing angle $|\theta|<0.01$ requires that $x / v \gtrsim 6$ and $M_{Z_{2}}>550 \mathrm{GeV}$ for $v / \bar{v}>5$. However, the detailed analysis is beyond the scope of the present article and we will only comment on this point in relation to the radiative symmetry breaking later.

\section{Scalar Potential and RGE Study}

We now study the symmetry breaking of this model at the low energy region. The second extra $U(1)_{y_{E}^{\prime}}$ is assumed to be broken at the intermediate scale $M_{R}$ so that we shall not consider it in the following study. This treatment will be justified because of this extra $U(1)$ can be decoupled not to induce any influence to the results in this section.

As is well-known, the large Yukawa couplings are essential for the study of the radiative symmetry breaking. Thus in addition to the usual top Yukawa coupling $h^{333} Q_{3} \bar{Q}_{3} H_{3}$, the largest Yukawa couplings $k^{333} S_{3} g_{3} \bar{g}_{3}$ and $\lambda^{333} S_{3} \bar{H}_{3} H_{3}$ of the extra colored fields and the Higgs fields to the singlet $S_{3}$ will be important in the analysis of the present model. Here we assume $k^{333}>k^{3 i j}$ and $\lambda^{333}>\lambda^{3 i j}(i, j \neq 3)$. Other terms can be neglected safely. The relevant terms in eq.(1) to the following investigation of the radiative symmetry breaking are

$$
W=h Q H \bar{T}+\lambda S \bar{H} H+k S g \bar{g}
$$


where we abbreviated the generation indices. The soft supersymmetry breaking terms corresponding to eq.(12) are

$$
-\mathcal{L}_{\mathrm{soft}}=\sum_{i} m_{i}^{2}\left|z_{i}\right|^{2}-\left(A_{h} h Q H \bar{T}+A_{\lambda} \lambda S \bar{H} H+A_{k} k S g \bar{g}+\text { h.c. }\right) .
$$

The first terms are the mass terms of the scalar components of all chiral superfields which are represented by $z_{i}$. The remaining terms are the trelinear couplings between the corresponding scalar components. We also introduce the gaugino mass terms $\sum_{a} M_{a} \bar{\lambda}_{a} \lambda_{a}$ where $a(=E, 1,2,3)$ specifies the gauge group. We do not ask the origin of these soft supersymmetry breaking terms here. Using eqs.(7),(12) and (13), the tree-level neutral Higgs scalar potential can be written as

$$
\begin{aligned}
V_{0} & =\frac{1}{8}\left(g_{2}^{2}+g_{1}^{2}\right)\left(v^{2}-\bar{v}^{2}\right)^{2}+\frac{1}{2} g_{E}^{2}\left(\frac{3}{2 \sqrt{6}} \bar{v}^{2}+\frac{1}{\sqrt{6}} v^{2}-\frac{5}{2 \sqrt{6}} x^{2}\right)^{2} \\
& +\lambda^{2} \bar{v}^{2} x^{2}+\lambda^{2} v^{2} x^{2}+\lambda^{2} \bar{v}^{2} v^{2} \\
& +m_{\bar{H}}^{2} \bar{v}^{2}+m_{H}^{2} v^{2}+m_{S}^{2} x^{2} \\
& -2 A_{\lambda} \lambda \bar{v} v x,
\end{aligned}
$$

where other charged fields are assumed not to have the VEVs.

Before our detailed study of this model we comment on a phenomenological feature which can be found without the potential minimization. Using this potential the neutral Higgs mass matrix can be written down. Noting that the smallest eigenvalue of the matrix is smaller than the smallest diagonal component, we can find the tree level upper bound of the lightest neutral Higgs mass. It is expressed as

$$
m_{h^{0}} \leq m_{Z}^{2}\left[\cos ^{2} 2 \beta+\frac{2 \lambda^{2}}{g_{1}^{2}} \sin ^{2} \theta_{W} \sin ^{2} 2 \beta+4 \sin ^{2} \theta_{W}\left(\frac{1}{\sqrt{6}} \sin ^{2} \beta+\frac{3}{2 \sqrt{6}} \cos ^{2} \beta\right)^{2}\right]
$$

where $\tan \beta=v / \bar{v}$. The first two terms of RHS of eq.(15) correspond to the bound which is derived from the usually studied extend model with a gauge singlet[26]. It is remarkable that the additional term raises the bound to some extent even at the tree level. The excess, which is due to the existence of the extra $U(1)$ gauge symmetry, is one of the typical features of our models. 1

\footnotetext{
${ }^{7}$ As suggested in ref.[27], on the argument of the lightest Higgs mass in the model with singlet fields it should be noted that the next-to-lightest Higgs might be the first observed one in future experiments if the lightest Higgs is singlet-dominated.
} 
Now we begin on the RGE study of this potential. The potential minimization conditions for $\bar{v}, v$ and $x$ are

$$
\begin{aligned}
&-\frac{1}{2}\left(g_{2}^{2}+g_{1}^{2}\right)\left(\bar{v}^{2}-v^{2}\right) \bar{v}+\frac{1}{2} g_{E}^{2} \frac{3}{2 \sqrt{6}}\left(\frac{3}{2 \sqrt{6}} \bar{v}^{2}+\frac{1}{\sqrt{6}} v^{2}-\frac{5}{2 \sqrt{6}} x^{2}\right) \bar{v} \\
&+2 \lambda^{2} \bar{v} v^{2}+2 m_{\bar{H}}^{2} \bar{v}-2 A_{\lambda} \lambda v x=0,=0 \\
& \frac{1}{2}\left(g_{2}^{2}+g_{1}^{2}\right)\left(\bar{v}^{2}-v^{2}\right) v+\frac{1}{2} g_{E}^{2} \frac{1}{\sqrt{6}}\left(\frac{3}{2 \sqrt{6}} \bar{v}^{2}+\frac{1}{\sqrt{6}} v^{2}-\frac{5}{2 \sqrt{6}} x^{2}\right) v \\
&+2 \lambda^{2} \bar{v}^{2} v+2 m_{H}^{2} v-2 A_{\lambda} \lambda \bar{v} x=0, \\
&-\frac{1}{2} g_{E}^{2} \frac{5}{2 \sqrt{6}}\left(\frac{3}{2 \sqrt{6}} \bar{v}^{2}+\frac{1}{\sqrt{6}} v^{2}-\frac{5}{2 \sqrt{6}} x^{2}\right)+2 m_{S}^{2}-2 A_{\lambda} \lambda \bar{v} v=0 .
\end{aligned}
$$

$\bar{v}$ and $v$ should satisfy the constraints for the weak boson mass

$$
m_{W}^{2}=\frac{1}{2} g_{2}^{2}\left(\bar{v}^{2}+v^{2}\right) \simeq(80 \mathrm{GeV})^{2}, \quad m_{Z_{1}}^{2} \simeq(91 \mathrm{GeV})^{2}
$$

at the potential minimum. We also have to check whether the charged Higgs scalars have non-vanishing VEVs. The necessary condition for the charge conservation is

$$
m_{2}^{2}+\lambda^{2} x^{2}+\frac{g_{2}^{2}}{4}\left(\bar{v}^{2}+v^{2}\right)+\frac{g_{1}^{2}}{4}\left(v^{2}-\bar{v}^{2}\right)+\frac{g_{E}^{2}}{\sqrt{6}}\left(\frac{3}{2 \sqrt{6}} \bar{v}^{2}+\frac{1}{\sqrt{6}} v^{2}\right)>0 .
$$

Generally $x$ is quite large as required from the neutral current phenomena in the present models. Therefore this condition is automatically satisfied in the most preferable parameter region. Under these conditions we numerically solve these coupled equations (16) whose coefficients are improved by the one-loop RGEs. For simplicity, we took the supersymmetry breaking scale $M_{S}$ as $m_{Z}$ so that our RGEs are supersymmetric in all energy range from a unification scale $M_{X}$ to $m_{Z}$.

In order to set up the initial conditions of the RGEs for the gauge coupling constants we have to estimate the unification scale $M_{X}$. The present model has three generations of the complete $27 \mathrm{~s}$ of $E_{6}$ as the massless matter contents. Therefore the one-loop $\beta$ function coefficient of the $S U(3)$ gauge coupling is $b_{3}=0$. As a result, the gauge coupling unification scale of $S U(3)$ and $S U(2)$ becomes $M_{X} \sim 10^{21} \mathrm{GeV}$, which is greater than the string scale $M_{\text {str }} \sim 4 \times 10^{17} \mathrm{GeV} 28$. However, this discrepancy can be consistent if we take account of the string threshold correction caused by the string heavy modes. As a

\footnotetext{
${ }^{8}$ We must consider the possibility of the color breaking too. We checked whether the conditions given in ref. [8] are satisfied. However, We should note that it has been suggested by many authors that the conditions are neither necessary nor sufficient in a certain cases.
} 
typical example, let us consider the case of the overall modulus $T$. The running coupling constant taking account of the string threshold correction can be written as [29]

$$
\frac{1}{g_{a}^{2}(\mu)}=\frac{k_{a}}{g_{\mathrm{str}}^{2}}-\frac{1}{16 \pi^{2}}\left(b_{a}^{\prime}-k_{a} \delta_{G S}\right) \log \left[\left(T+T^{*}\right)|\eta(T)|^{4}\right]+\frac{1}{16 \pi^{2}} b_{a} \log \left(\frac{M_{\mathrm{str}}}{\mu}\right)^{2}
$$

where $b_{a}^{\prime}=b_{a}+2 \sum_{i} T_{a}\left(C_{i}\right)\left(1+n_{i}\right) . \delta_{G S}$ is a duality anomaly cancellation coefficient due to the Green-Schwarz mechanism and $\eta(T)$ is a Dedekind function. $n_{i}$ is an integer called as the modular weight of a chiral superfield $C_{i} . T_{a}\left(C_{i}\right)$ is a second order index of the field $C_{i}$ with respect to the gauge group $G_{a}$. The unification condition for $S U(3)$ and $S U(2)$ is $k_{3} g_{3}^{2}\left(M_{X}\right)=k_{2} g_{2}^{2}\left(M_{X}\right)$. Taking the level $k_{3}=k_{2}=1$ as usual, this unification condition becomes

$$
\left(\frac{M_{\text {str }}}{M_{X}}\right)^{2}=\left(\left(T+T^{*}\right)|\eta(T)|^{4}\right)^{\frac{b_{3}^{\prime}-b_{2}^{\prime}}{b_{3}-b_{2}}} .
$$

Here it is remarkable that $\left(T+T^{*}\right)|\eta(T)|^{4}$ is less than one for any value of $T$. If we consider the model in which all the modular weights of the massless matter fields are $n_{i}=-1$, we get $b_{a}^{\prime}=b_{a}$ and $M_{\text {str }}<M_{X}$ can always be possible as suggested in ref. 16. In fact, in the present case $R e T \simeq 18$ makes the values of $M_{X}$ and $M_{\text {str }}$ consistent. On the basis of this argument we put the boundary conditions of the RGEs for the gauge coupling constants at the unification scale $M_{X}$ as

$$
g_{3}^{2}\left(M_{X}\right)=g_{2}^{2}\left(M_{X}\right)=\frac{5}{3} g_{1}^{2}\left(M_{X}\right)=\frac{5}{3} g_{E}^{2}\left(M_{X}\right)=g_{U}^{2}
$$

The boundary conditions for Yukawa couplings and the soft supersymmetry breaking parameters are set up at $M_{\text {str }}$ in the universal way as usual,

$$
\begin{aligned}
& \quad h\left(M_{\text {str }}\right)=h_{U}, \lambda\left(M_{\text {str }}\right)=\lambda_{U}, k\left(M_{\text {str }}\right)=k_{U}, \\
& m_{Q}^{2}\left(M_{\text {str }}\right)=m_{\bar{U}}^{2}\left(M_{\text {str }}\right)=m_{\bar{D}}^{2}\left(M_{\text {str }}\right)=m_{H}^{2}\left(M_{\text {str }}\right)=m_{\bar{H}}^{2}\left(M_{\text {str }}\right) \\
& \quad=m_{L}^{2}\left(M_{\text {str }}\right)=m_{N}^{2}\left(M_{\text {str }}\right)=m_{\bar{E}}^{2}\left(M_{\text {str }}\right)=m_{S}^{2}\left(M_{\text {str }}\right)=m_{0}^{2}, \\
& A_{h}\left(M_{\text {str }}\right)=A_{\lambda}\left(M_{\text {str }}\right)=A_{k}\left(M_{\text {str }}\right)=\nu m_{0}, \\
& M_{3}\left(M_{\text {str }}\right)=M_{2}\left(M_{\text {str }}\right)=M_{1}\left(M_{\text {str }}\right)=M_{E}\left(M_{\text {str }}\right)=m_{1 / 2} .
\end{aligned}
$$

These conditions for the soft supersymmetry breaking parameters are satisfied in the certain types of superstring models. In fact as an interesting one there is a case called as the dilaton dominated supersymmetry breaking 30. The large radius limit Calabi-Yau 
compactification also reveals the similar structure. In those cases the parameter space is largely restricted as $m_{1 / 2}=\sqrt{3} m_{0}$ and $\nu=\sqrt{3}$.

Starting from these boundary values at $M_{\text {str }}$ we will solve the full RGEs. The free parameters of our models at $M_{\text {str }}$ are the soft supersymmetry breaking parameters $m_{0}, \nu$, $m_{1 / 2}$ and Yukawa couplings $h_{U}, \lambda_{U}, k_{U}$. This parameter space can be somehow narrowed by imposing the additional constraints. To satisfy the experimental lower bound of the gluino mass, we require the following condition on the range of gaugino mass $m_{1 / 2}$ at the string scale,

$$
40 \mathrm{GeV} \leq m_{1 / 2} \leq 200 \mathrm{GeV}
$$

where the upper bound is set up on the basis of the naturalness consideration[31]. Also we set $\nu=\sqrt{3}$, for simplicity. However, it should be noted that this setting corresponds to the case of dilaton-dominated SUSY breaking which is favorable for the suppression of the EDMN as mentioned before. Using these boundary values we execute the numerical analysis following the previously mentioned procedure.

As an important input we set up the top quark mass in the range

$$
150 \mathrm{GeV} \leq m_{t} \leq 190 \mathrm{GeV}
$$

and search the parameter space, for which the top quark mass is in this region. As a result, we find that the radiative symmetry breaking can occur successfully in the rather wide parameter region. The values of Yukawa couplings at $M_{\text {str }}$ should be in the following range,

$$
\begin{gathered}
0.15 \leq h_{U} \leq 0.25 \\
0.3 \leq \lambda_{U} \leq 0.9 \\
0.1 \leq k_{U} \leq 0.9 .
\end{gathered}
$$

$h_{U}$ is confined in smaller region than other Yukawa couplings because the mass of top quark should be in the above range. The lower bounds of $\lambda_{U}$ and $k_{U}$ come from the requirement that the favorable symmetry breaking at weak scale occurs. Their upper bounds are related with the fact that $\langle S\rangle$ should not be generated at a very higher scale than $\langle S\rangle$ for the consistency of the analysis.?

\footnotetext{
${ }^{9}$ For more detailed analysis of this point the study based on the one-loop effective potential may be necessary 32 .
} 
For the phenomenological purpose we reconstruct our results as the relation between the physical particles masses, that is, the extra $Z$ boson mass and the top quark mass. Setting the top quark mass in the above range (25), for the various set of parameters we plot the corresponding extra $Z$ boson mass in Fig.2. The comments on its qualitative features are ordered. As easily understand, the large top mass region corresponds to the part of the large $h_{U}$ in the parameter space. Although we have not shown explicitly in figure, we find that the large values of $\lambda_{U}$ and $k_{U}$ have the tendency to bring the plotted points upward. This is based on the fact that the large $\lambda_{U}$ and $k_{U}$ make the singlet mass $m_{S}^{2}$ small through the RGE of $m_{S}^{2}$. This tendency of $k_{U}$ is more conspicuous than one of $\lambda_{U}$. The lower bound of $m_{Z_{2}}$ appears associated with the lower bounds of $\lambda_{U}$ and $k_{U}$. In this analysis we require that $\left|m_{Z}-m_{Z_{1}}\right| \leq 1 \mathrm{GeV}$ where $m_{Z}^{2} \equiv \frac{1}{2}\left(g_{1}^{2}+g_{2}^{2}\right)\left(v_{1}^{2}+v_{2}^{2}\right)$. If $m_{Z_{2}}$ is too small, $m_{Z_{1}}$ can not satisfy this condition. Our present requirement is not so quantitative one and this lower bound should not be taken seriously. As mentioned in the last part in sec.2, we need more detailed analysis by taking account of the constraints from the mixing angle and the radiative correction effects in order to estimate the lower bound of $m_{Z_{2}}$. However, we can make its rough estimation based on the experimental result on the mixing angle $\theta<0.01$ and the value of $1.4<\tan \beta<2.1$ which is the result of the present RGE study. In fact, we can read off the lower bound of $m_{Z_{2}}$ from Fig.1 as $290 \mathrm{GeV}<m_{Z_{2}}<420 \mathrm{GeV}$. The upper bound of the extra $Z$ boson mass can be read off as

$$
m_{Z_{2}} \leq 2000 \mathrm{GeV}
$$

This upper bound is crucially related with the upper bound of the soft breaking parameters $m_{0}$ and $m_{1 / 2}$. The result is not so sensitive to the values of $\nu$. We should recognize that the bound in eq. (26) is based on our setting of $m_{0}$ and $m_{1 / 2}$. Generally $m_{0}$ and $m_{1 / 2}$ increase, $m_{Z_{2}}$ becomes larger. In order to show this feature we show the $m_{0}$ dependence of the extra gauge boson mass $m_{Z_{2}}$ in Fig.3. As shown in this figure, if the Yukawa couplings $\lambda_{U}, k_{U}$ and $h_{U}$ are fixed, the relation between $m_{Z_{2}}$ and $m_{0}$ is almost linear. The termination of these lines at the certain values of $m_{0}$ represents that the condition (17) is no longer satisfied. Also $k_{U}$ is restricted when $\lambda_{U}$ and $h_{U}$ are fixed. For instance, In the case presented in Fig.3, the radiative symmetry breaking can not occur successfully when $k_{U}$ exceeds 0.6. The preferable range of $k_{U}$ depends on the value of $\lambda_{U}$. We find that the large $\lambda_{U}$ pushes up the allowed range of $k_{U}$ higher. For example, if $\lambda_{U}=0.7$, the available 
range of $k_{U}$ is $0.6<k_{U}<0.9$. Even if we take account of the $m_{0}$ dependence of $m_{Z_{2}}$, Fig. 3 shows that the upper bound of $m_{Z_{2}}$ presented above means to be reasonable from the viewpoint of the naturalness. Anyway, this bound seems to be interesting enough that such extra $Z$ boson may be found in the future collider.

As we mentioned before, the extra color triplets $g$ and $\bar{g}$ can not acquire the large mass at the sufficiently high energy scale in the present model. However, they can be heavy enough not to be detected directly in the current experiment because their mass originate from the VEV of the singlet field $S$. T From the present analysis of the radiative symmetry breaking we can estimate the mass of the fermionic components of such extra color triplets. We plot it against the extra $Z$ boson mass in Fig.4. Because the dominant source of the extra $Z$ boson mass is also the VEV of the singlet filed $S$, they are related linearly and the steepness depends on the coupling $k$. The phenomenology of these extra color triplets is one of the interesting aspects of our model, although it is beyond the scope of the present paper. Their detail property will depend on the structure of the discrete symmetry as discussed in ref. [14]. Similar phenomena suggested there will also be expected in our model.

\section{Summary}

We examined the radiative symmetry breaking in a model with a low energy extra $U(1)$ symmetry. We stressed that the introduction of the extra $U(1)$ s to the MSSM has some preferable features for the explanation of the $\mu$-problem and the neutrino mass. It may be a very promising extension of the MSSM. Such models are severely restricted by the theoretical and phenomenological requirements. In this paper we proposed a superstring inspired $E_{6}$ model as such a favorable example. We showed that in our model the radiative symmetry breaking of $S U(2) \times U(1)$ occurs successfully in a certain parameter region. For such a region the $\mu$-problem is solved dynamically and the top quark mass can be situated in the region where the data obtained recently suggest. The extra $Z$ boson mass is also predicted in relation to the top quark mass. The upper bound of this extra $Z$ boson mass

\footnotetext{
${ }^{10}$ In principle, the gauge invariant coupling $\mathcal{N} \bar{D} g$ can be included in the superpotential. However, If such coupling exists, $\langle\mathcal{N}\rangle \neq 0$ causes an extremely large mixing between $\bar{D}$ and $g$ and our model becomes unrealistic. Therefore we must assume that such a term is forbidden by a suitable discrete symmetry in the present model.
} 
is $m_{Z_{2}} \leq 2000 \mathrm{GeV}$, which may be encouraging for us to find it in the future collider. This model also yields the large right-handed neutrino mass without introducing the large scale supersymmetry breaking. Thus it can give the small neutrino mass which is appropriate to the MSW solution for the solar neutrino problem. Its relation to the inflation scenario is also very interesting. This issue will be treated elsewhere[33].

\section{Acknowledgement}

The authors thank S. Matsumoto for helpful discussions. The work of D. S. is supported in part by a Grant-in-Aid for Scientific Research from the Ministry of Education, Science and Culture (\#05640337 and \#06220201). 


\section{References}

[1] J. Ellis, S. Kelley and D. V. Nanopoulos, Phys. Lett. B249(1990)441; Phys. Lett. B260(1991)131.

P. Langacker, M. Luo, Phys. Rev. D44(1991)817.

V. Amaldi, W. de Boer and H. Furstenau, Phys. Lett. B260(1991)447.

[2] W. Hollik, Plenary talk at the XVI Int. Symp. on Lepton-Photon Interactions, Cornell Univ., Ithaka, NY. USA (Aug. 10-15, 1993).

[3] CDF collaboration,preprint FERMILAB-PUB-94/097-E, $\mathrm{CDF} / \mathrm{PUB} / \mathrm{TOP} / \mathrm{PUBLIC} / 2561$.

[4] K. Inoue, A. Kakuto, H. Komatsu and S. Takeshita, Prog. Theo. Phys. 68(1982)927.

L. Alvarez-Gaume, J. Polchinski and M. B. Wise, Nucl. Phys. B221(1983)495.

J. Ellis, D. V. Nanopoulos and K. Tamvakis, Phys. Lett. B121(1983) 123.

J. Ellis, J. Hagelin, D. V. Nanopoulos and K. Tamvakis, Phys. Lett. B125(1983)275.

L. Ibañéz and C. López, Phys. Lett. B126(1983)54.

[5] J. E. Kim and H. P. Nilles, Phys. Lett. B138 (1984)150.

[6] J. E. Kim and H. P. Nilles, Phys. Lett. B263(1991)79.

E. J. Chun, J. E. Kim and H. P. Nilles, Nucl. Phys. B370(1992) 105.

J. A. Casas and C. Muñoz, Phys. Lett. B306(1993)228.

[7] G. F. Giudice and A. Masiero, Phys. Lett. B206 (1988)480.

[8] J. -P. Derendinger and C. A. Savoy, Nucl. Phys. B237 (1984)307.

[9] H. P. Nilles, M. Srednicki and D. Wyler, Phys. Lett. B120(1983)364.

L. E. Ibáñez and J. Mas, Nucl. Phys. B286(1987)107.

J. Ellis, J. F. Gunion, H. E. Haber, L. Roszkowski and F. Zwirner, Phys. Rev. D39(1989)844. 
[10] GALLEX Collab., P. Anselmann et al., Phys. Lett. B285(1992)376, and references therein.

[11] M. B. Green, J. H. Schwarz and E. Witten, Superstring Theory, Cambridge University Press (1987).

[12] M. Dine, V. Kaplunovsky, M. Mangano, C. Nappi and N. Seiberg, Nucl. Phys. B259(1985)549.

[13] T. Matsuoka and D. Suematsu, Nucl. Phys. B274 (1986)106; Prog. Theor. Phys. 76(1986)886.

[14] D. J. Castaño and S. P. Martin, preprint MIT-CTP-2345, NUB-3097-94-TH (hepph/9408230).

[15] C. A. Lütkin and G. G. Ross, Phys. Lett. B214(1988)357.

P. Zoglin, Phys. Lett. B228(1988)47.

C. Hattori, M. Matsuda, T. Matsuoka and H. Mino, Prog. Theor. Phys. 82(1989)599.

[16] A. Brignole, L.E.Ibáñez and C.Muñoz, Nucl. Phys. B422(1994)125.

[17] P. Zoglin, Phys. Lett. B243(1990)215.

[18] M. Gell-Mann, P. Ramond and S. Slansky, in Supergravity, eds. P. van Nieuwenhuizen and D. Freedman (North-Holland, Amsterdam, 1979)p315.

T. Yanagida, in Proc. Workshop on Unified Theory and Baryon Number in the Universe, eds. A. Sawada and H. Sugawara(KEK, Tsukuba, Japan, 1979).

[19] S. P. Mikheyev and A. Yu. Smirnov, Yad. Fiz. 42(1985)[Sov. J. Nucl. Phys. 42(1985)913.]

L. Wolfenstein, Phys. Rev. D20(1979)2634.

[20] M. Cvetič and P. Langacker, Phys. Rev. D46(1992)R2759,

N. Haba, C. Hattori, M. Matsuda, T. Matsuoka and D. Mochinaga, Prog. Theor. Phys. 92(1994)153.

[21] K. Choi, Phys. Rev. Lett. 72(1994)1592. 
[22] T. Matsuoka, H. Mino, D. Suematsu, S. Watanabe, Prog. Theor. Phys. 76(1986)915. F. Zwirner, Int. J. Mod. Phys. A3(1988)49.

J. Hewett and T. G. Rizzo, Phys. Lett. 183C(1989)193.

[23] K. Choi and J.E. Kim, Phys. Lett. 165B(1986)901.

T. Matsuoka, D. Suematsu, Prog. Theor. Phys. 76(1986)901.

[24] J. Ellis, K. Enqvist, D. V. Nanopoulos and F. Zwirner, Nucl. Phys. B276(1986)14.

[25] L3 Collaboration, Phys. Lett. B306(1993)187, DELPHI Collaboration, preprint CERN-PPE/94-121.

[26] M. Drees, Int. J. Mod. Phys. A4(1989)3635.

[27] J-I. Kamoshita, Y. Okada, M. Tanaka Phys. Lett. B328(1994) 67.

[28] V. S. Kaplunovsky, Nucl. Phys. B307(1988)145.

[29] L. Dixon, V. S. Kaplunovsky and J. Louis, Nicl. Phys. B355(1991)649. J.-P. Derendinger, S. Ferrara, C. Kounnas and F. Zwirner, Nucl. Phys. B372 (1992) 145 .

I. Antoniadis, K. Narain and T. Taylor, Phys. Lett. B267(1991)37.

[30] V. Kaplunovsky and J. Louis, Phys. Lett. B306(1993)269, R. Barbieri, J. Louis and M. Moretti, Phys. Lett. B312(1993)451.

[31] J. Ellis, K. Enqvist, D. V. Nanopoulos and F. Zwirner, Mod. Phys. Lett. A1( 1986) 57.

R. Barbieri and G. Giudice, Nucl. Phys. B306(1988)63.

[32] G. Gamberini, G. Ridolfi, F. Zwirner, Nucl. Phys. B331(1990) 331.

[33] D. Suematsu and Y. Yamagishi, in preparation. 


\section{Table1}

Particle contents in 27 of $E_{6}$ and extra $U(1)$ charge assignments. $\mathcal{N}$ has the same charges as $N$.

\begin{tabular}{c|cccc}
$\psi$ & $\left(S U(3)_{C}, S U(2)_{L}\right)$ & $U(1)_{y}$ & $U(1)_{y_{E}}$ & $U(1)_{y_{E^{\prime}}}$ \\
\hline \hline$Q$ & $(3,2)$ & $1 / 6$ & $-1 / 2 \sqrt{6}$ & $\sqrt{10} 0 / 12$ \\
$\bar{U}$ & $\left(3^{*}, 1\right)$ & $-2 / 3$ & $-1 / 2 \sqrt{6}$ & $\sqrt{10} 0 / 12$ \\
$\bar{D}$ & $\left(3^{*}, 1\right)$ & $1 / 3$ & $-1 / \sqrt{6}$ & $-\sqrt{10} / 6$ \\
$L$ & $(1,2)$ & $-1 / 2$ & $-1 / \sqrt{6}$ & $-\sqrt{10} / 6$ \\
$\bar{E}$ & $(1,1)$ & 1 & $-1 / 2 \sqrt{6}$ & $\sqrt{1} 0 / 12$ \\
$H$ & $(1,2)$ & $1 / 2$ & $1 / \sqrt{6}$ & $-\sqrt{1} 0 / 6$ \\
$\bar{H}$ & $(1,2)$ & $-1 / 2$ & $3 / 2 \sqrt{6}$ & $\sqrt{10} 0 / 12$ \\
$N$ & $(1,1)$ & 0 & 0 & $\sqrt{10} / 3$ \\
$S$ & $(1,1)$ & 0 & $-5 / 2 \sqrt{6}$ & $\sqrt{10} 0 / 12$ \\
$g$ & $(3,1)$ & $-1 / 3$ & $1 / \sqrt{6}$ & $-\sqrt{10} / 6$ \\
$\bar{g}$ & $\left(3^{*}, 1\right)$ & $1 / 3$ & $3 / 2 \sqrt{6}$ & $\sqrt{10} 0 / 12$
\end{tabular}




\section{Figure Captions}

\section{Fig.1}

The contours of the extra $Z$ boson mass $M_{Z_{2}}=300,400,500,600 \mathrm{GeV}$ (solid lines), the

mixing angle $\theta=0.03,0.02,0.01$ (dashed-doted lines) and $\Delta=0.015,0.006,0.004,0.002$ (doted lines) in the $(\bar{v} / v)-(x / v)$ plane.

\section{Fig.2}

The extra $Z$ boson mass corresponding to the top quark mass. Points are plotted for each values of $h_{U}$. The values of $k_{U}$ and $\lambda_{U}$ are not explicitly presented in this figure.

\section{Fig.3}

The dependence of the extra $Z$ boson mass on the soft supersymmetry breaking scalar mass $m_{0}$ where we take $\lambda_{U}=0.5$ and $h_{U}=0.2$.

\section{Fig.4}

The mass of the extra colored fermion $g, \bar{g}$ corresponding to the extra $Z$ boson mass. Points are plotted for under each values of $k_{U}$. The values of $k_{U}$ and $\lambda_{U}$ are not explicitly presented in this figure. 
This figure "fig1-1.png" is available in "png" format from: http://arxiv.org/ps/hep-ph/9411239v2 


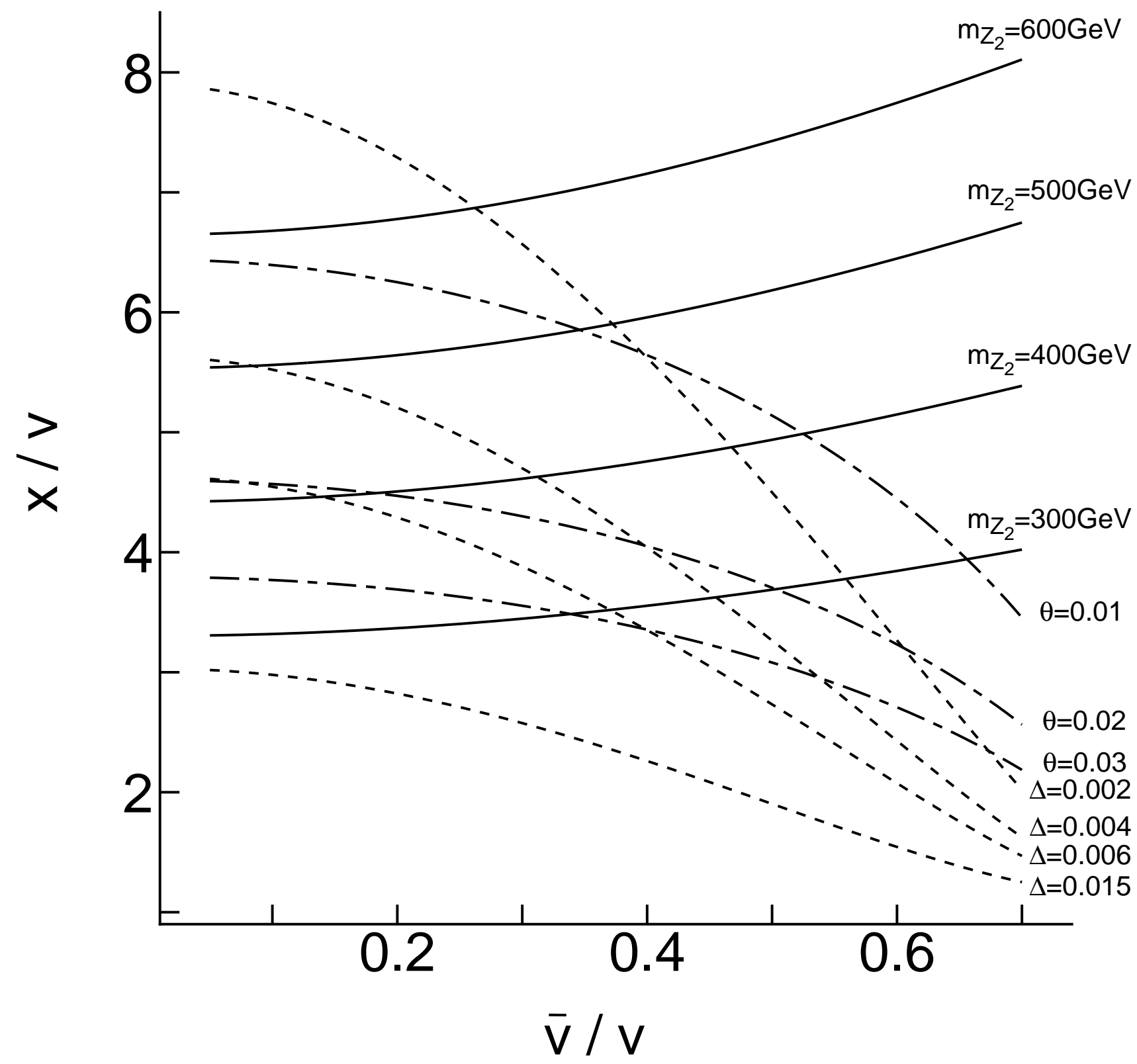

Fig.1 
This figure "fig1-2.png" is available in "png" format from: http://arxiv.org/ps/hep-ph/9411239v2 


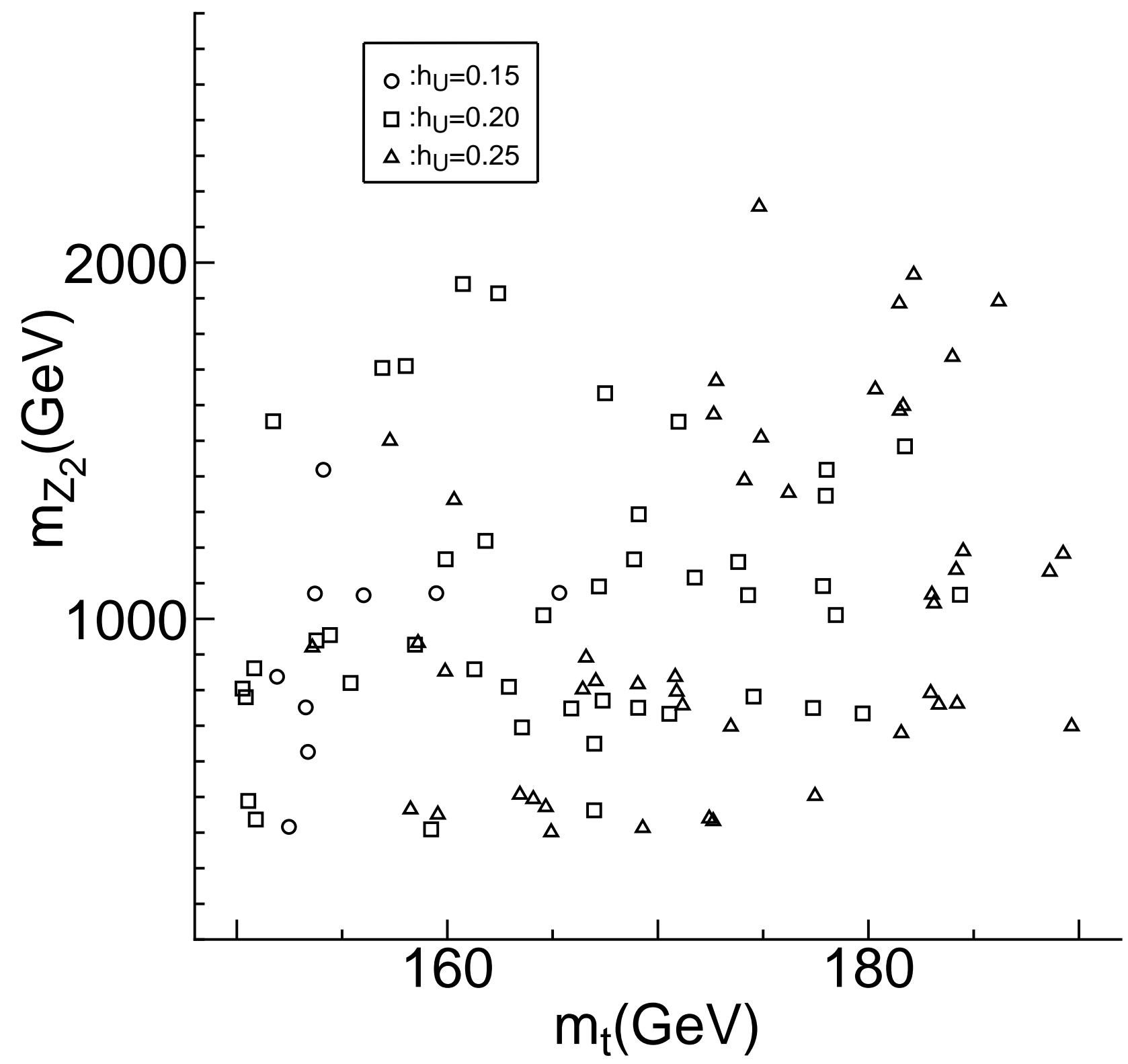

Fig.2 
This figure "fig1-3.png" is available in "png" format from: http://arxiv.org/ps/hep-ph/9411239v2 
$\lambda_{U}=0.5, h_{U}=0.2$

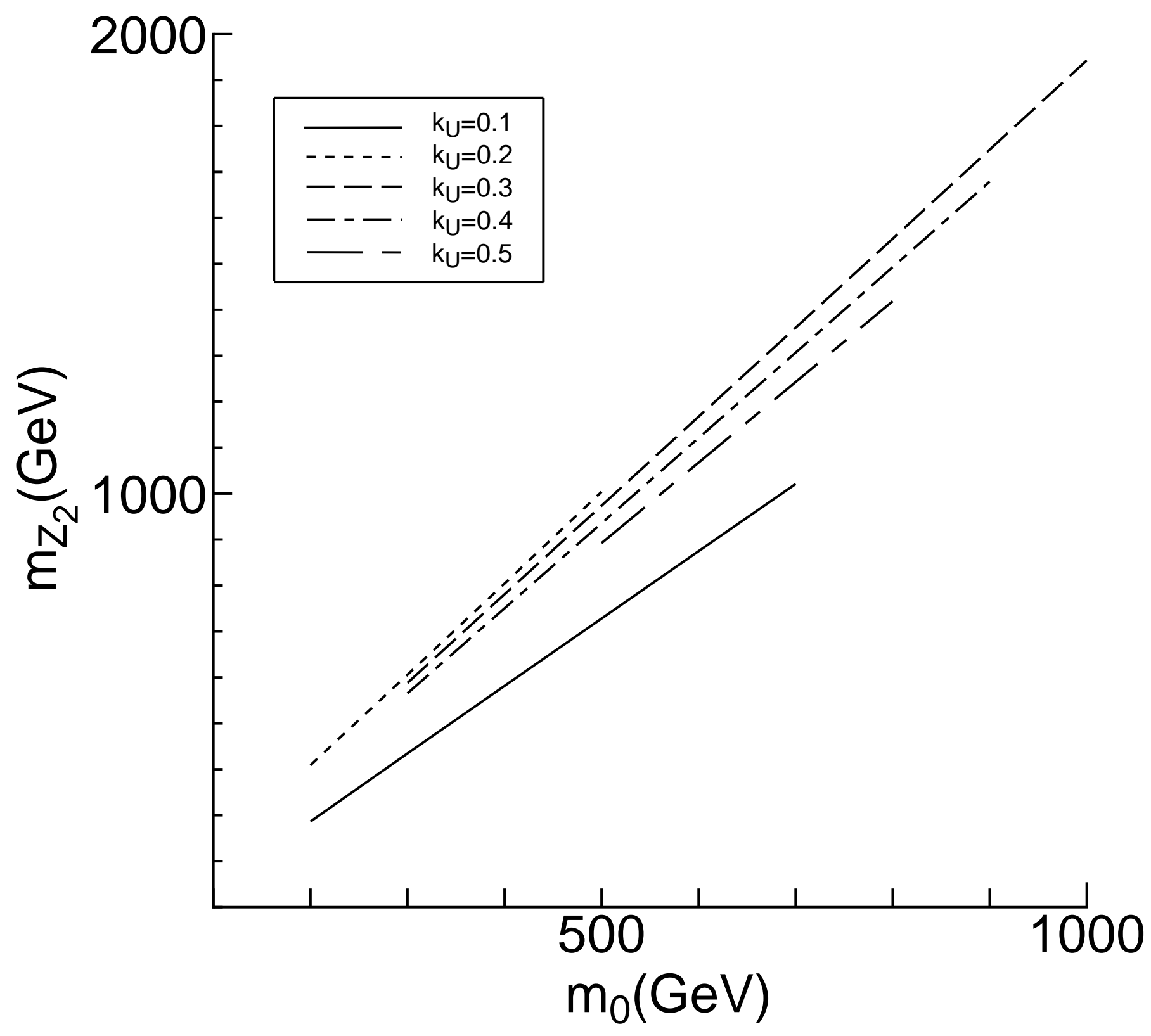

Fig.3 
This figure "fig1-4.png" is available in "png" format from: http://arxiv.org/ps/hep-ph/9411239v2 


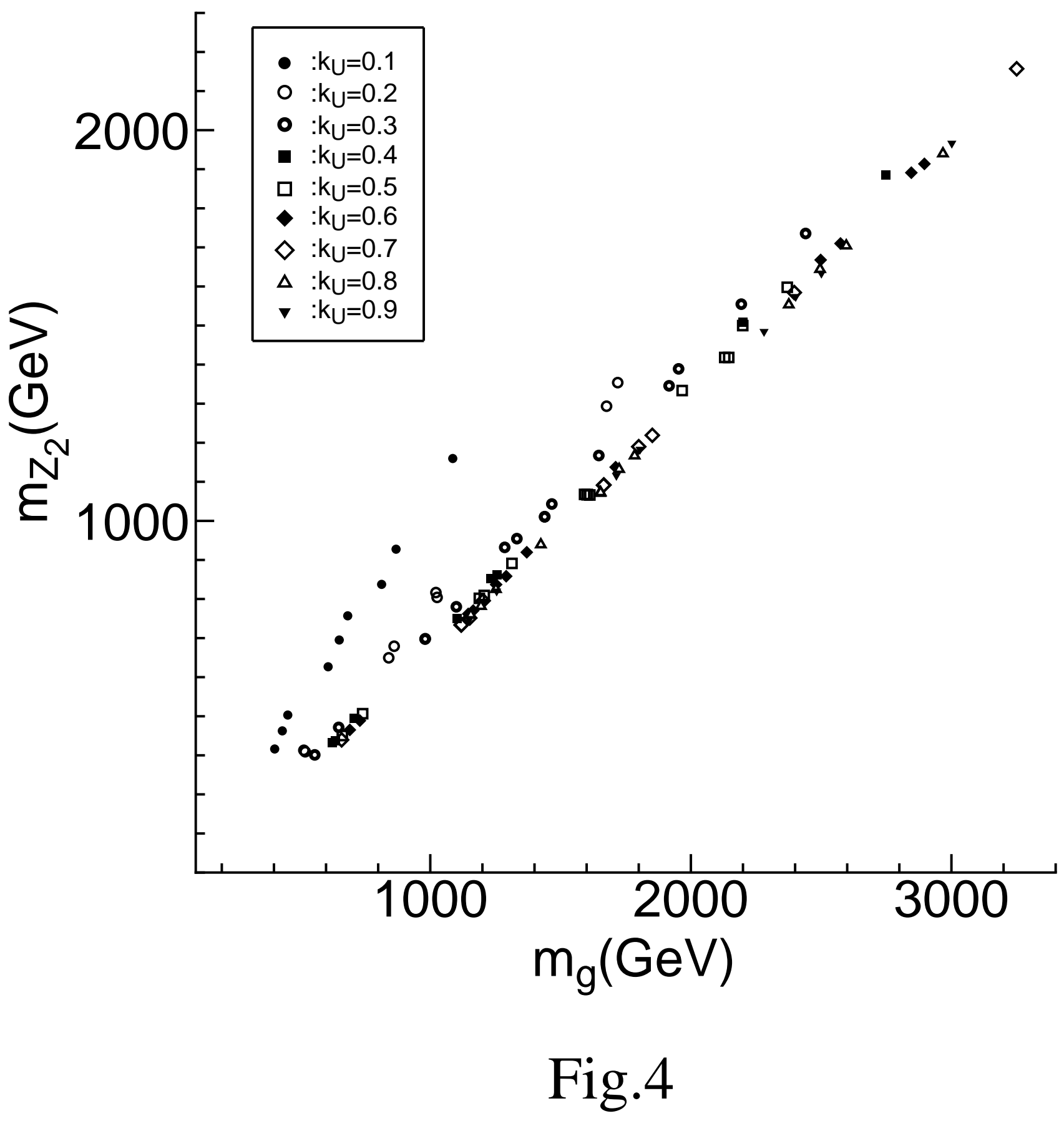

